\title{
EL VaR PARA LA GESTIÓN DEL RIESGO DEL PORTAFOLIO DE INVERSIONES
}

\author{
THE VAR FOR THE RISK MANAGEMENT OF THE \\ INVESTMENT PORTFOLIO
}

Pedro Pablo Chambi Condori Universidad Nacional Jorge Basadre Grohmann Tacna, Perú

ORCID: http://orcid.org/0000-0002-2565-2780 Correo electrónico: pchambic@unjbg.edu.pe

\section{RESUMEN}

Objetivo: Analizar cómo la rentabilidad diaria, la varianza de cada uno de los componentes de una cartera de inversiones y la correlación entre los activos de la cartera tienen incidencia en el VaR del portafolio de inversiones. Método: Es un tipo de investigación descriptiva, explicativa con enfoque cuantitativo; ya que tiene como base la cuantificación del valor promedio y del VaR de la cartera de inversiones. La muestra seleccionada por convención estuvo conformada por 8 títulos que cotizan en el mercado de valores del Perú, de los cuales, se seleccionó 3 activos de renta variable con los que se ha conformado una cartera de inversiones de renta variable, con los datos de cotización diaria desde enero 2014 hasta diciembre del 2017. Resultados: EI VaR del portafolio y el valor promedio de la cartera se obtienen mediante el método de simulación de Montecarlo para un escenario de variación de rentabilidad promedio diaria del $10 \%$, se constata que la volatilidad de la cartera tiene incidencia directa en el VaR y la rentabilidad influye en el VaR de manera directa. Conclusiones: Aplicando la teoría de diversificación de Harry Markowitz y la simulación de Montecarlo, los resultados obtenidos muestran el efecto de la variación de rentabilidad y la volatilidad en el valor del VaR y sobre el valor promedio del portafolio.

Palabras clave: Portafolio; inversiones; simulación; riesgo.

\begin{abstract}
Objective: Analyze how the daily profitability, the variance of each of the components of an investment portfolio and the correlation between the portfolio assests have an incidence in the VaR of the investment portfolio. Method: It is a type of quantitative, descriptive, explanatory with a quantitative approach; that have as a based on the quantification of the average value and the $\mathrm{VaR}$ of the investment portfolio. The sample selected by convention was conform by 8 titles that listed on the Peruvian Securities Market, of which, was selected 3 equities assets with which formed a portfolio of equity investments, with the daily quoted data from January 2014 to December 2017. Results: The VaR of the portfolio and the average value of the portfolio are obtained by applying the MonteCarlo simulation for a variation scenario of average daily profitability of $10 \%$, it is verified that the volatility of the portfolio has a direct incidence on the VaR and the profitability influence in the VaR directly. Conclusions: Applying the Harry Markowitz diversification theory and the MonteCarlo simulation, the results obtained prove the effect of the variation in profitability and volatility on the VaR value and on the average value of the portfolio.
\end{abstract}

Keywords: Portfolio; investments; simulation; risk.

(c) Los autores. Este artículo es publicado por la Revista Quipukamayoc, Universidad Nacional Mayor de San Marcos. Este es un artículo de acceso abierto, distribuido bajo los términos de la Licencia Creative Commons Atribución-NoComercial-Compartirlgual 4.0 Internacional.(http://creativecommons.org/licenses/by-nc-sa/4.0/), que permite el uso no comercial, distribución y reproducción en cualquier medio, siempre que la obra original sea debidamente citadas. 


\section{INTRODUCCIÓN}

Debido a los niveles de volatilidad que ocurren en diversos mercados financieros de renta variable como resultado de choques económicos, financieros, sociales y ambientales; para los inversionistas es de singular importancia estudios de simulación del VaR de portafolio de inversiones a diferentes niveles de confianza estadística a fin de prever recaudos y coberturas necesarias ante los niveles de probabilidad de pérdida esperada. La cuantificación de pérdida esperada de portafolios de inversión a determinados niveles de confianza estadística es de suma importancia para los agentes involucrados, a fin de analizar diversos escenarios riesgosos para gestionar de la mejor forma sus posiciones en el mercado de valores de activos financieros de renta variable. El método de simulación de Montecarlo permite estimar el VaR esperado ante la variación simulada de las variables aleatorias que intervienen en el modelo, que, en el caso en estudio, son la rentabilidad media diaria, la volatilidad de la misma y el nivel de correlación entre los activos que intervienen en la cartera, el proceso de simulación de Montecarlo tiene la secuencia que se inicia con la obtención del modelo, seguido por la identificación de las variables aleatorias, luego, la identificación del tipo de distribución aleatoria que describe cada uno de las variables identificadas en el paso previo y, finalmente, la especificación de la simulación.

Sobre el tema de gestión de riesgos financieros utilizando el VaR, se tiene las referencias que se desarrollan en líneas siguientes:

El VaR, cuantifica la pérdida máxima esperada (o la peor pérdida) sobre un horizonte de tiempo objetivo dentro de un intervalo de confianza estadística. La medición del $\mathrm{VaR}$ requiere la fijación del tiempo y el nivel de confianza estadística (Jorion, 2010).

Venegas (2007) afirma que, "bajo el supuesto de normalidad en el rendimiento de un portafolio de activos, es posible encontrar expresiones sencillas de VaR de un portafolio de inversiones" (p.696).

Machain (2014), expresa que:

Una de las maneras de estimar el VaR es el enfoque paramétrico, que se basa en el análisis de varianzas y covarianzas de las variables. El VaR permite cuantificar la pérdida esperada de una cartera frente a determinados niveles de volatilidad de las variables de entrada del modelo (...). Para la estimación del VaR, es la simulación de Montecarlo, la cual da una mayor flexibilidad a la hora de establecer los supuestos. En su forma más primitiva, el VaR por Montecarlo asume que los retornos se distribuyan normalmente y que la matriz de varianzas y covarianzas capture todas las dependencias posibles entre los activos que componen la cartera (p.419).

El VaR es ampliamente utilizado en las instituciones financieras en la medición de riesgo de mercado y riesgos operativos a $95 \%$ y $99 \%$ de confianza y para un horizonte de tiempo de uno a diez días. El método de Montecarlo es un nombre bastante general para cualquier enfoque de medición de riesgo que implique la simulación de un modelo paramétrico explícito para cambios de factores de riesgo. De este modo, el método puede ser condicional o incondicional, dependiendo de si es que el modelo adoptó un modelo de serie temporal dinámico para cambios de factores de riesgo o un modelo de distribución estática. El primer paso para la simulación es la obtención del modelo paramétrico, en segundo lugar, consiste en identificar las variables de riesgo que involucra el modelo, y, en tercer término, identificar el tipo de distribución aleatoria que tiene cada una de las variables de riesgo identificadas en el modelo que ha de utilizarse para la ejecución de la simulación (McNeil, Frey y Embrechts, 2005).

Gutiérrez (2018), sostiene que, "la aleatoriedad en las variables de entrada causa la variabilidad en las variables de resultado" (p.27).

Taco (2017), refiere que:

La simulación por el método de Montecarlo es un modelo que se basa en el muestreo aleatorio $y$ repetido y en el análisis estadístico para calcular los resultados. Es muy semejante a la técnica de diseño de experimentos, en cuanto a su finalidad, ya que el resultado específico no se conoce de antemano, pero se puede predecir por medio de una simulación (p.8).

Markowitz (1959), para la organización de una cartera de inversiones, hace referencia a la teoría de estructuración de carteras de inversión que están expresadas en las ecuaciones que se enuncian en líneas siguientes:

Rentabilidad diaria de un activo de renta variable:

$R_{t}=\frac{P_{t}-P_{t-1}+D_{t}}{P_{t-1}}$, en donde:

$\mathrm{R}_{\mathrm{t}}$ : rentabilidad esperada, $\mathrm{P}_{\mathrm{t}}$ : precio del activo en el tiempo t. $\mathrm{P}_{\mathrm{t}-1}$ : precio del activo del periodo anterior. $\mathrm{D}_{\mathrm{t}}$ : dividendos en el periodo.

Que luego la rentabilidad se puede expresar mediante la ecuación:

$R_{i}=100 * \ln \left(\frac{P_{t}}{P_{t-1}}\right)$, en donde: 
$\mathrm{R}_{\mathrm{i}}$ : Rentabilidad del activo en el tiempo t. $\mathrm{P}_{\mathrm{t}}$ : precio del activo en el tiempo t. $\mathrm{P}_{\mathrm{t}-1}$ : precio del activo en el tiempo t-1 y el valor de 100 es constante que denota los valores en $100 \%$.

La rentabilidad media de la cartera de inversiones está expresada por la ecuación siguiente:

$E(R)=W_{a}{ }^{\star} E\left(R_{a}\right)+W_{b}{ }^{\star} E\left(R_{b}\right)+\cdots+W_{n}{ }^{\star} E\left(R_{n}\right)$ en donde:

$E 囚$ : rentabilidad media de la cartera de inversiones.

$W_{a}$ : proporción del activo a, $W_{b}$ : proporción del activo b, $W_{n}$ : proporción del activo $\mathrm{n}$.

$E\left(R_{a}\right)$ : rentabilidad media del activo a, $E\left(R_{b}\right):$ rentabilidad del activo b, $E\left(R_{n}\right)$ : rentabilidad media del activo n.

Como $E(R)$, es una medida de posición central, por tanto su medida de dispersión es la desviación estándar, que es la expresión siguiente:

$\sigma=\sqrt{\left(W_{a}^{2}\right) * \sigma_{a}^{2}+2 * W_{a} W_{b} \operatorname{Cov}\left(R_{a}, R_{b}\right)+\cdots+\left(W_{n}^{2}\right) \sigma_{n}^{2}}$,

en donde $W_{a}, W_{b} y W_{n}$ : son los pesos de los componentes de la cartera de inversiones. Las $\sigma \_\mathrm{a}^{\wedge} 2, \ldots, \sigma \_\mathrm{n}^{\wedge} 2$ : varianza de cada uno de componentes del portafolio.

Valor en Riesgo (VaR)

$V a R_{p}=Z * I * \sigma * \sqrt{\Delta t}$,

En donde, Z: es el valor a un determinado nivel de confianza, I: Inversión en el portafolio de inversión, $\sigma$ es el riesgo de la cartera, $\Delta \mathrm{t}$ es la variación de tiempo.

A continuación, se expresa la pregunta principal de la investigación:

¿De qué manera el efecto variación en rentabilidad, varianza y correlación entre los activos que conforman la cartera tienen incidencia en el VaR de un portafolio de inversiones?

El estudio tuvo como hipótesis central, la variación de rentabilidad y la volatilidad en el valor del VaR tiene incidencia sobre el valor promedio de las inversiones, mientras que el objetivo general consiste en analizar cómo la rentabilidad diaria, la varianza de cada uno de los componentes de una cartera de inversiones y la correlación entre los activos de la cartera tiene incidencia en el VaR del portafolio de inversiones. El primer objetivo específico consiste en aplicar la simulación del método de Montecarlo sobre el modelo VaR del portafolio de inversiones para estimar la pérdida potencial esperada que puede ocurrir en una cartera de inversiones que está conformada por activos de renta variable y el segundo objetivo específico, está orientada a aplicar la simulación de Montecarlo sobre el modelo de estructuración de carteras de inversión para la obtención del valor promedio de una cartera de inversiones.

Se delinea como hipótesis de investigación, a los cambios que ocurren con la rentabilidad diaria, la volatilidad que éstos tienen en el tiempo y la correlación entre los componentes de la cartera, tienen incidencia en el VaR del portafolio de inversiones.

\section{MATERIAL Y MÉTODOS}

La investigación es de tipo descriptiva y explicativa, en la que se ha desarrollado un análisis cuantitativo. Como materiales de investigación se pueden mencionar a la cotización diaria al cierre de los activos que conforman la muestra, los que se recogen del repositorio de la Bolsa de Valores de Lima, del periodo de enero 2014 a diciembre del 2017. Por convención, se ha seleccionado 8 activos de renta variable que cotizan en el mercado de valores del Perú, de cuya cantidad se eligen a 3 activos con las que se conforma una cartera de inversiones, los activos seleccionados son los que presentan las más altas rentabilidades promedio diaria y correlación baja entre ellos, con lo cual se obtiene la rentabilidad media diaria de la cartera y su respectivo riesgo.

Para cada activo, se registra la cotización diaria desde enero 2014 hasta diciembre 2017, con cuya data se obtiene la rentabilidad diaria, utilizando la ecuación siguiente:

$R_{i}=100 * \ln \left(\frac{P_{t}}{P_{t-1}}\right)$,

El portafolio de inversiones, como se hizo notar en líneas arriba, se conforma con tres activos, con cuyos valores se calcula la rentabilidad media diaria, utilizando la ecuación siguiente:

$$
E(R)=W_{a}{ }^{\star} E\left(R_{a}\right)+W_{b}{ }^{\star} E\left(R_{b}\right)+\cdots+W_{n}{ }^{\star} E\left(R_{n}\right)
$$

Con esta ecuación se obtiene la rentabilidad de la cartera de inversiones y luego el valor promedio del portafolio, que resulta de aplicar la ecuación:

Valor promedio de la cartera $=$ Inversión $^{\star}(1+$ rentabilidad media diaria esperada).

Y posteriormente se obtiene el modelo paramétrico del VaR, cuya ecuación es el que se presenta a continuación:

$V a R_{p}=Z * I * \sigma * \sqrt{\Delta t}$, en donde:

Z: es el valor a un determinado nivel de confianza, I: Inversión en el portafolio de inversión, $\sigma$ es el riesgo de la cartera, $\Delta \mathrm{t}$ tiempo en el que se estima el VaR. 
Ya construido el modelo del VaR de la cartera de inversión en el ambiente de Excel, el proceso ilustrativo que sigue es el que se puede observar en la figura 1, proceso que se sigue con el soporte del software Risk simulator, generando 10 mil interacciones de simulación del VaR (ver figura 1).

El proceso que se presenta en la ilustración antecedente, comprende el circuito desarrollado para el proceso de simulación del VaR del portafolio de inversiones con activos de renta variable.

Finalmente, se aplica la simulación de Montecarlo sobre el modelo VaR, teniendo como variables aleatorias de entrada a la rentabilidad media diaria y el riesgo de cada uno de los activos que conforman la cartera en mención y la correlación entre los activos de la cartera, generando una muestra de 10 mil valores de VaR, como variable de salida, que luego mediante el tratamiento estadístico respectivo se obtienen los resultados descriptivos e inferenciales que se presentan a continuación.

\section{RESULTADOS}

Se obtiene como resultados descriptivos la rentabilidad media diaria y la volatilidad de cada uno de los activos de la muestra. Del examen de los valores de rentabilidad media diaria de cada uno de los activos de la muestra, se puede observar, que el activo del sector financiero Credicorp es el que presenta la más alta rentabilidad, en segundo lugar, el activo del sector de maquinarias y equipos pesados Ferreycorp; y en tercer orden, el activo del sector construcción Cemento Pacasmayo.

Tal cual sugiere la teoría de conformación de carteras de inversión con activos de renta variable de Markowitz(1959), la selección de activos sigue el criterio de elegir a aquellos activos que tienen la mayor rentabilidad, y por otro lado, que expresen entre sí menores correlaciones, como resultado del procedimiento seguido en la construcción de la cartera de inversión, se tiene la matriz de correlación entre los activos de la muestra, que en este orden del caso, el portafolio de inversión queda conformada por los activos: Credicorp, Ferreycorp y Cemento Pacasmayo (ver tablas 1 y 2 ).

Por otro lado, es importante observar de manera ilustrativa el comportamiento histórico de la rentabilidad diaria de cada uno de los activos que han sido considerados como muestra para la experiencia en cuestión, tal como ya se ha afirmado en líneas arriba, de esta muestra se extraen los

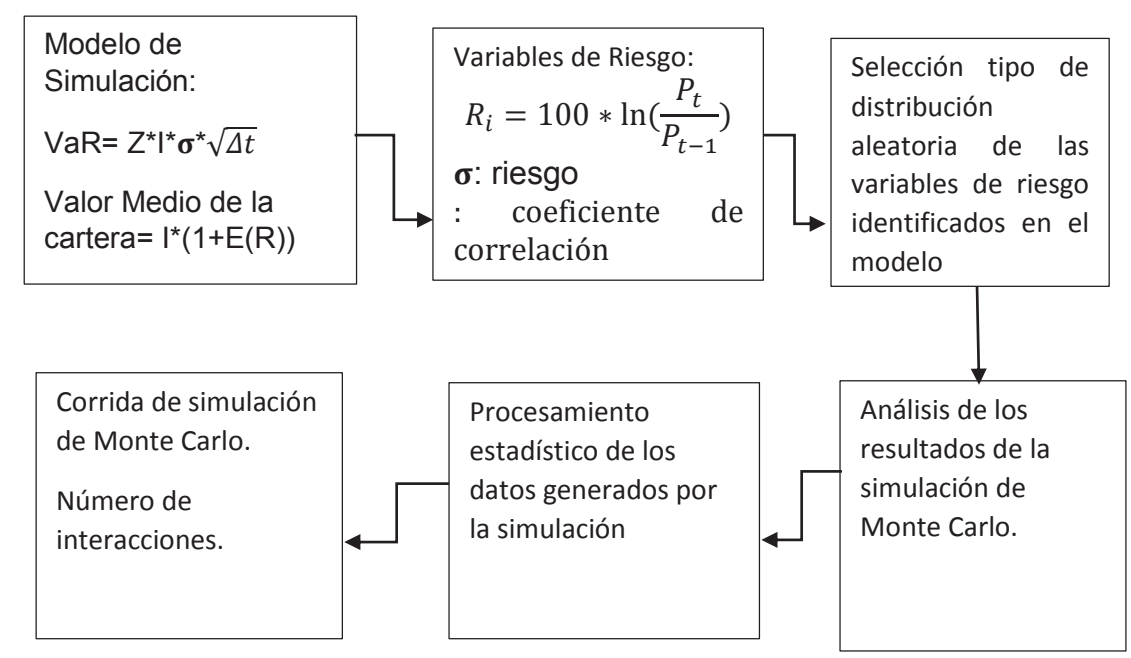

Figura 1. Proceso de simulación y análisis de riesgo.

Fuente: Risk Analysis in Investment Appraisa, Savvides (1994).

Tabla 1

Rentabilidad media diaria y desviación estándar de los activos de la muestra.

\begin{tabular}{ccccccccc}
\hline Indicador & Alicorp & Ferreycorp & Volcan & $\begin{array}{c}\text { Cemento } \\
\text { Pacasmayo }\end{array}$ & Credicorp & $\begin{array}{c}\text { Cemento } \\
\text { Andina }\end{array}$ & $\begin{array}{c}\text { Buena } \\
\text { ventura }\end{array}$ & Inter-corp \\
\hline Rentabilidad media & 0,015 & 0,032 & 0,009 & 0,026 & 0,047 & $-0,023$ & 0,021 & 0,022 \\
Desviación estándar & 1,355 & 12,266 & 3,027 & 1,548 & 1,537 & 1,758 & 3,253 & 1,189 \\
\hline
\end{tabular}

Fuente: Repositorio de la Bolsa de Valores de Lima. 
Tabla 2

Matriz de correlación de los activos de la muestra - Bolsa de Valores de Lima - Perú.

\begin{tabular}{lllllllll}
\hline \multicolumn{1}{c}{ Activos } & \multicolumn{1}{c}{ Alicorp } & Buena-ventura & $\begin{array}{c}\text { Candi } \\
\text { Na }\end{array}$ & Pacasmayo & Credicorp & Ferreycorp & Intercorp & Vol-can \\
\hline Alicorp & 1 & 0,111 & 0,293 & 0,241 & 0,258 & 0,139 & 0,125 & 0,235 \\
Buenaventura & 0,111 & 1 & 0,108 & 0,096 & 0,242 & 0,056 & 0,076 & 0,229 \\
Candina & 0,293 & 0,108 & 1 & 0,338 & 0,235 & 0,198 & 0,104 & 0,297 \\
Cpacasmayo & 0,241 & 0,096 & 0,338 & 1 & 0,181 & 0,188 & 0,065 & 0,254 \\
Credicorp & 0,258 & 0,242 & 0,235 & 0,181 & 1 & 0,150 & 0,037 & 0,280 \\
Ferreycorp & 0,139 & 0,056 & 0,198 & 0,188 & 0,150 & 1 & 0,060 & 0,186 \\
Intercorp & 0,125 & 0,076 & 0,104 & 0,065 & 0,037 & 0,060 & 1 & 0,025 \\
Volcan & 0,235 & 0,229 & 0,297 & 0,254 & 0,280 & 0,186 & 0,025 & 1 \\
\hline
\end{tabular}

Fuente: Repositorio de la Bolsa de Valores de Lima (2014-2017).
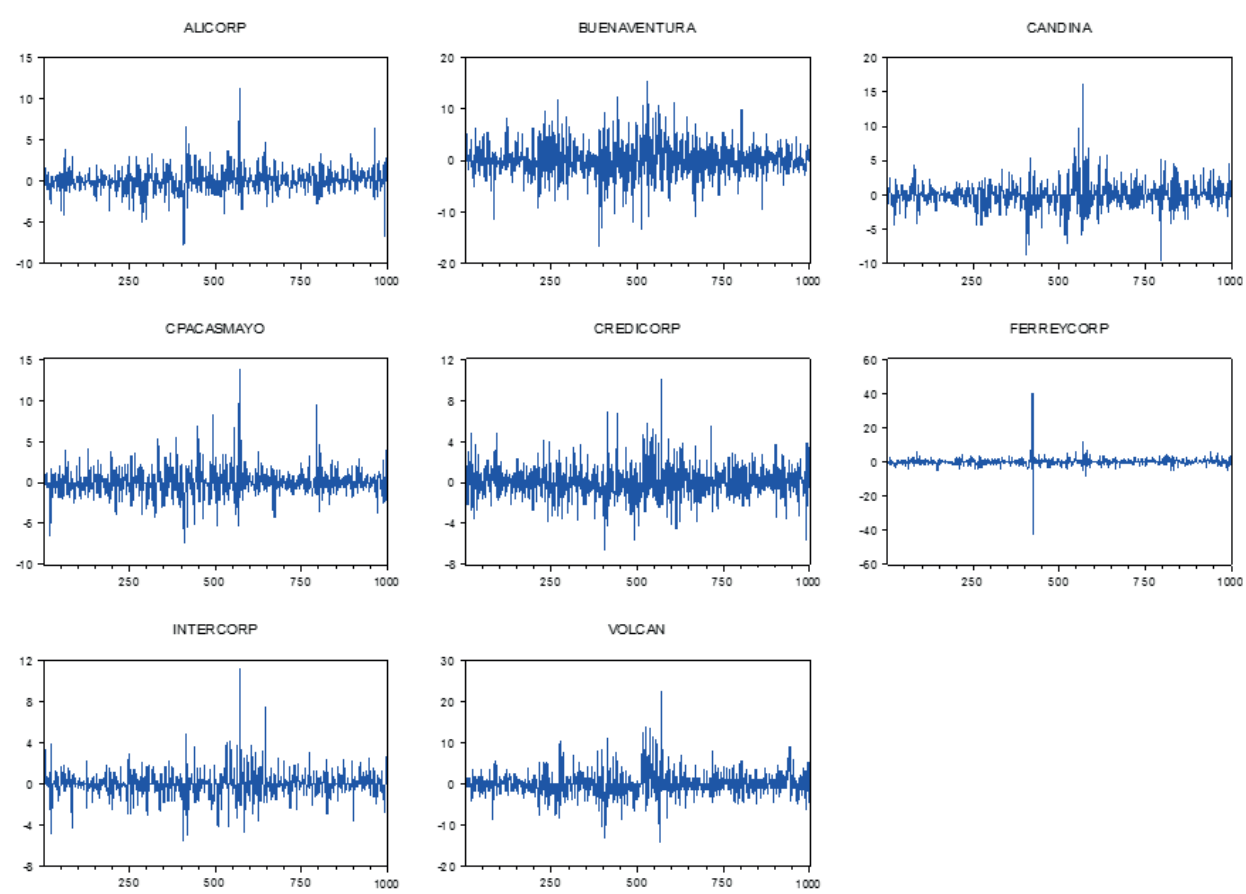

Figura 2. Serie de tiempo de rentabilidad diaria de los activos de la muestra analizada.

Fuente: Repositorio de la Bolsa de Valores de Lima.

activos para la conformación de la cartera que es utilizada para la experiencia en cuestión (ver figura 2).

Conformada la cartera de inversión, siguiendo la teoría de estructuración de carteras de inversión de Markowitz (1959), con la data histórica de rentabilidad diaria de los tres activos se estima en primera instancia, la rentabilidad diaria de cada uno de los activos la rentabilidad media de cada activo y consecuentemente su medida de dispersión que es la desviación estándar, para luego asumir los pesos de cada componente, cuyos resultados se han expuesto en líneas antecedentes (ver tabla 3).
Con los datos expuestos en la tabla antecedente, se construye el modelo de rentabilidad media del portafolio de inversiones y su medida de dispersión, el valor promedio del portafolio y el VaR de la cartera de inversión, valores obtenidos con los datos históricos, los indicadores presentes son: rentabilidad y el riesgo de la cartera, y por otro lado el valor promedio de la cartera y el VaR a 95\% de confianza (ver tabla 4).

La experiencia consistió en aplicar la simulación de Montecarlo sobre el modelo VaR al 5\%, teniendo como variables aleatorias de entrada, la rentabilidad y el riesgo, 
Tabla 3

Rentabilidad media diaria, volatilidad y pesos de los componentes de la cartera.

\begin{tabular}{lrrr}
\hline \multicolumn{1}{c}{ Indicador } & \multicolumn{1}{c}{ Ferreycorp } & Cemento Pacasmayo & \multicolumn{1}{c}{ Credicorp } \\
\hline Rentabilidad media diaria & 0,0315 & 0,0257 & 0,0469 \\
Desviación estándar & 12,2659 & 1,5476 & 1,5369 \\
Pesos & 0,1 & 0,3 & 0,6 \\
\hline
\end{tabular}

Fuente: Repositorio de la Bolsa de Valores de Lima: 2014-2017.

Tabla 4

Determinación del VaR del portafolio de Inversión.

\begin{tabular}{rr} 
Rentabilidad= & 0,039017345 \\
Riesgo= & 1,798378841 \\
Alfa & $5 \%$ \\
Fondos invertidos & 1000000 \\
Valor promedio cartera & 1039017,35 \\
Desvest en dinero & 17983,79 \\
Valor de corte & 1009436,65 \\
VaR al 5\% & 29580,70 \\
\hline
\end{tabular}

Fuente: Repositorio de la Bolsa de Valores de Lima (2014-2017).

Tabla 5

Resultados descriptivos de la simulación de Montecarlo de VaR.

\begin{tabular}{lrrr}
\hline \multicolumn{1}{c}{ Indicador } & \multicolumn{1}{c}{ VaR } & \multicolumn{1}{c}{ Rentabilidad } & Volatilidad \\
\hline Mean & 29574,43 & 0,039 & 1,798 \\
Median & 29574,51 & 0,039 & 1,798 \\
Maximum & 41317,05 & 0,054 & 2,512 \\
Minimum & 17293,48 & 0,023 & 1,051 \\
Std. Dev. & 2957,77 & 0,004 & 0,180 \\
Skewness & 0,00 & $-0,001$ & $-0,001$ \\
Kurtosis & 3,00 & 2,998 & 3,003 \\
Jarque-Bera & 0,00 & 0,004 & 0,004 \\
Probability & 1,00 & 0,998 & 0,998 \\
Sum & 295744296,27 & 390,00 & 17979,98 \\
Sum Sq. Dev. & 874554648,03 & 0,15 & 323,32 \\
Observations & 10000 & 10000 & 10000 \\
\hline
\end{tabular}

Fuente: Valores generados mediante la simulación de Montecarlo.

asumiendo para cada uno de ellos distribución aleatoria normal, variación de +-10\%, generando 10000 interacciones, obteniendo la misma cantidad de VaR, que en este caso es la variable de salida de la simulación, para luego con el soporte de la estadística descriptiva obtener los valores de rentabilidad y la volatilidad. El indicador de Jarque Bera en asimetría $=0$ y kurtosis $=3$, evidencia que lo datos con los que se ha obtenido estos resultados pertenecen a una distribución normal (ver tabla 5 y figura 3 ).

Los resultados de la simulación de VaR, presentados en la figura 3 , ilustran el valor medio, los valores mínimos y máximos a $95 \%$ de confianza, con una kurtosis $=3$ estadísticamente significativa y riesgo inducido de $10 \%$. 
Por otro lado, los resultados presentados en la figura 4, exhiben el VaR para cada nivel de percentil, observándose, valor ascendente de VaR para la evolución ascendente de percentil de volatilidad, lo que significa el $10 \%$ de probabilidad para VaR mayor que 33 364,41 soles (ver figura 4).

Del resultado de la simulación de Montecarlo, para el caso hipotético del importe de inversión en la cartera en análisis, ante la variación de la rentabilidad y la volatilidad en $+-10 \%$, tal cual se puede apreciar en la figura antecedente, en donde la media de la peor pérdida esperada es el valor de 29574,43 , y el 90\% de VaR se encuentra entre 25000 a
35000 soles, por otro lado, la probabilidad de peor pérdida esperada menor a 23773.96 soles es $2,5 \%$, la peor pérdida esperada mayor a 33364,41 soles tiene la probabilidad de $10 \%$ (ver figura 5).

La figura 5, muestra la simulación de Montecarlo de Valor Promedio de la cartera a diferentes niveles de percentil, el valor medio, mínimo y máximo.

Los resultados presentados en líneas antecedentes, muestran asimetría $=0$ y kurtosis $=3$, que denotan la procedencia de los datos de una muestra que tienen distribución normal (ver figura 5).

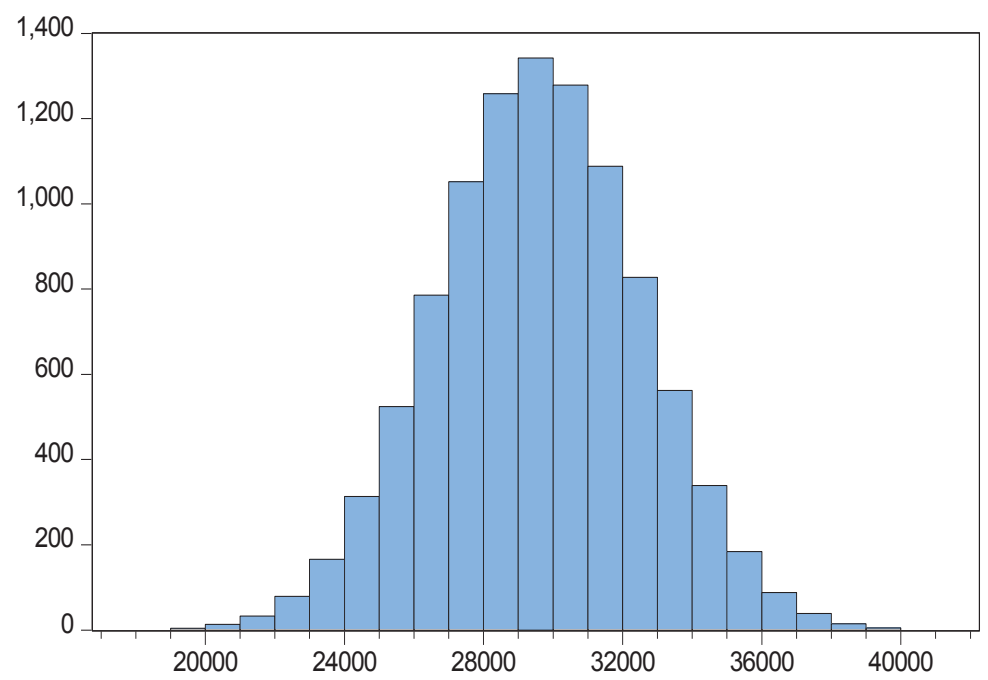

\begin{tabular}{|lr|}
\hline \multicolumn{2}{|l|}{ Series: VAR } \\
Sample 1 10000 \\
Observations & 10000 \\
& \\
Mean & 29574.43 \\
Median & 29574.51 \\
Maximum & 41317.05 \\
Minimum & 17293.48 \\
Std. Dev. & 2957.771 \\
Skewness & -0.000637 \\
Kurtosis & 3.002857 \\
& \\
Jarque-Bera & 0.004077 \\
Probability & 0.997964 \\
\hline
\end{tabular}

Figura 3. Valores descriptivos del VaR obtenido mediante Simulación de Montecarlo. Fuente: Elaboración Propia.

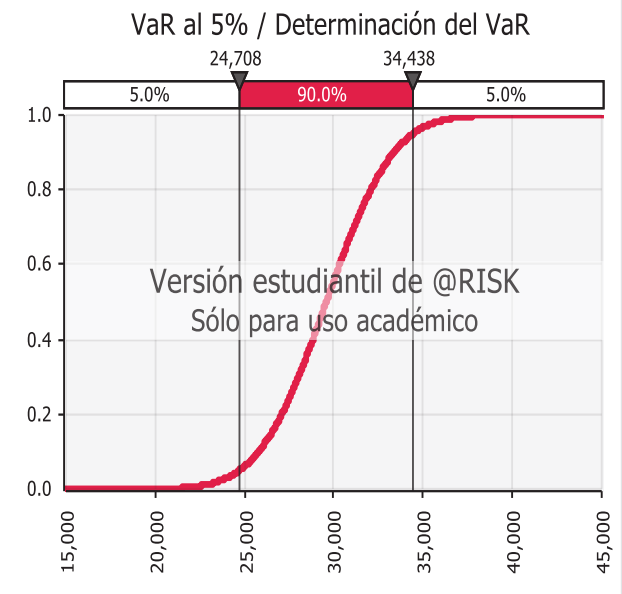

Figura 4. Resultado de simulación Montecarlo de VaR a 95\% de confianza.

\begin{tabular}{|c|c|c|c|}
\hline Estadísticos r & esumen para $V$ & al $5 \% / \mathrm{I}$ & ación del V \\
\hline Estadísticos & & reentil & VaR \\
\hline Mínimo & $17,293.48$ & $1.0 \%$ & $22,694.09$ \\
\hline Máximo & $41,317.05$ & $2.5 \%$ & $23,773.96$ \\
\hline Media & $29,574.43$ & $5.0 \%$ & $24,707.67$ \\
\hline Desv Est & $2,957.77$ & $10.0 \%$ & $25,783.06$ \\
\hline Varianza & $8,748,410.22$ & $20.0 \%$ & $27,084.46$ \\
\hline Indice de sesgo & 0.00 & $25.0 \%$ & $27,579.35$ \\
\hline Curtosis & 3.00 & $50.0 \%$ & $29,574.11$ \\
\hline Mediana & $29,574.11$ & $75.0 \%$ & $31,568.84$ \\
\hline Moda & $29,685.70$ & $80.0 \%$ & $32,063.42$ \\
\hline $\mathrm{X}$ izquierda & $24,707.67$ & $90.0 \%$ & $33,364.41$ \\
\hline P izquierda & 0.05 & $95.0 \%$ & $34,438.48$ \\
\hline $\mathrm{X}$ derecha & $34,438.48$ & $97.5 \%$ & $35,370.41$ \\
\hline$P$ derecha & 0.95 & $99.0 \%$ & $36,453.19$ \\
\hline Heromos & 000 & & \\
\hline
\end{tabular}

Fuente: Simulación generada con Risk Simulator 7.6 de Palisade. 
VALOR PROMEDIO CARTERA / Determinación de...

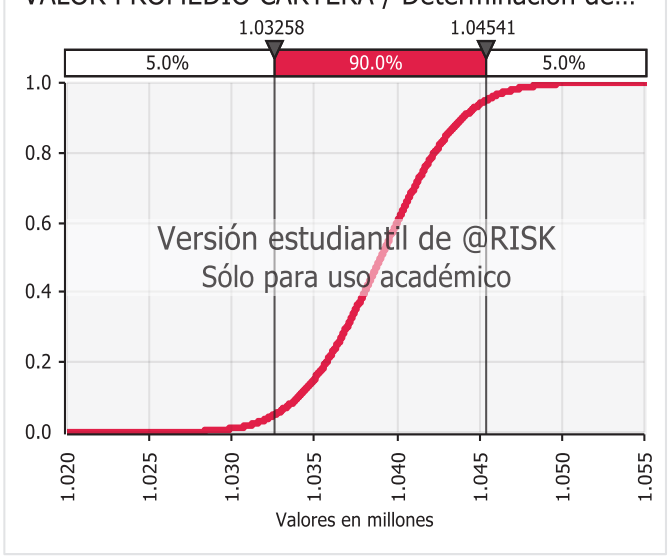

\begin{tabular}{l|r|r|r}
\hline \multicolumn{3}{|c}{ Estadísticos resumen para VALOR PROMEDIO CARTERA } \\
\hline Estadísticos & \multicolumn{3}{|c}{ Percentil } \\
\hline Mínimo & $1,021,436.94$ & $\mathbf{1 . 0} \%$ & $1,029,918.12$ \\
\hline Máximo & $1,053,540.53$ & $\mathbf{2 . 5 \%}$ & $1,031,354.14$ \\
\hline Media & $1,038,999.64$ & $\mathbf{5 . 0} \%$ & $1,032,584.45$ \\
\hline Desv Est & $3,900.72$ & $\mathbf{1 0 . 0} \%$ & $1,034,001.37$ \\
\hline Varianza & 15215652.87 & $\mathbf{2 0 . 0} \%$ & $1,035,716.78$ \\
\hline Indice de sesgo & -0.004359825 & $\mathbf{2 5 . 0 \%}$ & $1,036,369.48$ \\
\hline Curtosis & 3.009375796 & $\mathbf{5 0 . 0} \%$ & $1,038,999.20$ \\
\hline Mediana & $1,038,999.20$ & $\mathbf{7 5 . 0} \%$ & $1,041,630.36$ \\
\hline Moda & $1,038,951.09$ & $\mathbf{8 0 . 0} \%$ & $1,042,281.58$ \\
\hline X izquierda & $1,032,584.45$ & $\mathbf{9 0 . 0} \%$ & $1,043,996.39$ \\
\hline P izquierda & $5 \%$ & $\mathbf{9 5 . 0} \%$ & $1,045,414.89$ \\
\hline X derecha & $1,045,414.89$ & $\mathbf{9 7 . 5 \%}$ & $1,046,640.99$ \\
\hline P derecha & $95 \%$ & $\mathbf{9 9 . 0 \%}$ & $1,048,067.92$ \\
\hline \#Errores & 0 & & \\
\hline & & &
\end{tabular}

Figura 5. Valor promedio de la cartera.

Fuente: Simulación de Montecarlo con Risk Simulator, Palisade.

Tabla 6

Incidencia de rentabilidad y volatilidad en el VaR del portafolio.

\begin{tabular}{|c|c|c|c|c|}
\hline Dependent Variable: VAR & VaR & & & \\
\hline \multicolumn{5}{|l|}{ Method: Least Squares } \\
\hline \multicolumn{5}{|l|}{ Date: 07/20/19 Time: 07:35 } \\
\hline \multicolumn{5}{|l|}{ Sample: 110000} \\
\hline \multicolumn{5}{|c|}{ Included observations: 10000} \\
\hline Variable & Coefficient & Std. Error & t-Statistic & Prob. \\
\hline C & 12645,08 & 24,98 & 506,30 & 0,00 \\
\hline LOG(VOLATILIDAD) & 29116,45 & 21,21 & 1372,52 & 0,00 \\
\hline RENTABILIDAD & $-118,85$ & 551,10 & $-0,22$ & 0,83 \\
\hline R-squared & 0,99 & \multicolumn{2}{|c|}{ Mean dependent var } & 29574,43 \\
\hline Adjusted R-squared & 0,99 & \multicolumn{2}{|c|}{ S.D. dependent var } & 2957,77 \\
\hline S.E. of regression & 214,91 & \multicolumn{2}{|c|}{ Akaike info criterion } & 13,58 \\
\hline Sum squared resid & 461723634,82 & \multicolumn{2}{|c|}{ Schwarz criterion } & 13,58 \\
\hline Log likelihood & $-67890,07$ & \multicolumn{2}{|c|}{ Hannan-Quinn criter. } & 13,58 \\
\hline F-statistic & 941986,94 & \multicolumn{2}{|c|}{ Durbin-Watson stat } & 2,03 \\
\hline Prob(F-statistic) & 0,00 & & & \\
\hline
\end{tabular}

Fuente: Valores simulados con Risk Simulator, Palisade.

En el modelo obtenido mediante los datos generados por simulación de Montecarlo con diez mil valores simulados sobre el modelo del VaR, se puede apreciar que la volatilidad de la cartera tiene incidencia a elevar el riesgo de pérdida potencial (VaR) de la cartera, mientras que la rentabilidad tiene expresa una incidencia indirecta sobre el VaR del portafolio de inversión (ver tabla 6). El modelo que describe el comportamiento del VaR ante la presencia de volatilidad y rentabilidad, con significancia estadística, es el que se presenta en la siguiente expresión:
Ecuación 1:

$V a R=12645,08+29116,45 * \ln ($ volatilidad $)-118,85 *$ $($ Rentabilidad $)+e$

El modelo expresado en la ecuación 1, describe el efecto de las variables de entrada sobre el valor del VaR, con indicadores y coeficientes estadísticamente significativas, con los valores de DW=2,03, la prueba F del modelo y la prueba $\mathrm{T}$ de los coeficientes estadísticamente aceptables, 
queda demostrada la hipótesis de la investigación y logrado los objetivos de la investigación.

\section{DISCUSIÓN}

El proceso seguido en la medición del VaR mediante el método de Montecarlo, que, según McNeil et al. (2005) es utilizado por instituciones financieras en la cuantificación de riesgos operativos y riesgo de mercado, en el estudio en cuestión se utiliza para la medición del riesgo del portafolio de inversiones. Como afirma Gutiérrez (2018), la aleatoriedad de las variables de entrada, da como resultado la variabilidad de la variable resultado que en este caso es el VaR del portafolio que efectivamente, de cara a los resultados, una elevada volatilidad en la cartera de inversiones hace expansiva el valor del VaR, información muy importante para el gestor de carteras de inversiones y para los mismos inversionistas, que deben gestionar de manera óptima sus exposiciones al riesgo. La conformación de la cartera de inversión, mostrada en el estudio de acuerdo a la teoría de estructuración de carteras de Markowitz (1959), ha sido utilizada para la generación de los resultados del VaR en 10 mil interacciones, de cuyo procesamiento estadístico se obtienen los valores del VaR, rentabilidad y la volatilidad del portafolio. El objetivo general del estudio era analizar cómo la rentabilidad diaria, la varianza de cada uno de los componentes de una cartera de inversiones y la correlación entre los activos de la cartera tiene incidencia en el VaR del portafolio de inversiones, cuyos resultados se han expuesto en el estudio sugerido por Machain (2014) de la incidencia de las variables aleatorias de entrada sobre la variable de salida que es el $\mathrm{VaR}$, y en el mismo sentido han delineado McNeil et al. (2005). El estudio tuvo como hipótesis central mostrar la variación de rentabilidad y la volatilidad en el valor del VaR, que tiene incidencia sobre el valor promedio de las inversiones, en este extremo queda contrastada la hipótesis con los resultados obtenidos y presentados en la tabla 6 y expresado en la ecuación 1, resultados que tienen aproximación a lo afirmado por Gutiérrez (2018) quien indica que las variables de entrada provocan variabilidad en las variables de salida, que en este caso, son el VaR y el Valor promedio de la Cartera, en el caso específico de la experiencia, la variable rentabilidad tiene una relación directa sobre el VaR y la variable de riesgo tiene una relación indirecta sobre el VaR, en el mismo sentido de las afirmaciones de Venegas (2007) quien dice que, es posible encontrar expresiones sencillas de VaR de un portafolio de inversiones, mostrando qué variables son las que elevan y qué variables son las que minimizan el VaR, que es la peor pérdida esperada ante las fluctuaciones de sus variables de entrada.

\section{REFERENCIAS BIBLIOGRÁFICAS}

Gutiérrez, H. (2018). Manual de Análisis Cuantitativo del Riesgo e Introducción al Uso del Risk. Universidad de Lima, Facultad de Ciencias Empresariales y Económicas, Carrera de Negocios Internacionales, 7(5.1).

Jorion, P. (2010). Valor en Riesgo. México D.F.: Editorial Limusa.

Machain, L. (2014). Simulación de Modelos Financieros. Buenos Aires: Editorial Alfaomega.

McNeil, A., Frey, R. \& Embrechts, P. (2005). Quantitative Risk Management. New Jersey: Ediciones Princeton University.

Markowitz, H. (1959). Portfolio Selection: Efficient Diversification of Investment. New York, New Haven: Ediciones Yale University.

Savvides, S. (1994). Risk Analysis in Investment Appraisal. Cambridge, Massachusetts: Ediciones Harvard University.

Taco, D. (2017). Simulación de Montecarlo para la gestión de Costos en la Evaluación de Proyectos de Inversión, caso: Construcción del Hotel Wyndham Gran Cóndor PRONOBIS S.A. de Ecuador. Buenos Aires: Ediciones Universidad de Buenos Aires.

Venegas, F. (2007). Riesgos Financieros y Económicos. México DF.: Editorial Thomson. 
